\title{
Simulating Displacement and Velocity Signals by Piezoelectric Sensor in Vibration Control Applications
}

\author{
G. J. Sheu, ${ }^{1}$ S. M. Yang, ${ }^{2}$ and W. L. Huang ${ }^{3}$ \\ ${ }^{1}$ Department of Electrical Engineering, Hsiuping University of Science and Technology, Taichung, Taiwan \\ ${ }^{2}$ Department of Aeronautics and Astronautics, National Cheng Kung University, Tainan City 701, Taiwan \\ ${ }^{3}$ Department of Aeronautics and Astronautics, National Cheng Kung University and Mildex Corporation, Taiwan
}

Correspondence should be addressed to S. M. Yang, smyang@mail.ncku.edu.tw

Received 15 May 2012; Accepted 17 June 2012

Academic Editor: C. R. Bowen

Copyright ( $) 2012$ G. J. Sheu et al. This is an open access article distributed under the Creative Commons Attribution License, which permits unrestricted use, distribution, and reproduction in any medium, provided the original work is properly cited.

\begin{abstract}
Intelligent structures with built-in piezoelectric sensor and actuator that can actively change their physical geometry and/or properties have been known preferable in vibration control. However, it is often arguable to determine if measurement of piezoelectric sensor is strain rate, displacement, or velocity signal. This paper presents a neural sensor design to simulate the sensor dynamics. An artificial neural network with error backpropagation algorithm is developed such that the embedded and attached piezoelectric sensor can faithfully measure the displacement and velocity without any signal conditioning circuitry. Experimental verification shows that the neural sensor is effective to vibration suppression of a smart structure by embedded sensor/actuator and a building structure by surface-attached piezoelectric sensor and active mass damper.
\end{abstract}

\section{Introduction}

Composite structures with surface-mounted or -embedded piezoelectric materials as sensors and/or actuators have been investigated for they possess mechanical simplicity, efficient electromechanical energy conversion, and ability to integrate within structures. Much attention to date has been on analysis and experiment of active vibration control by using piezoelectric sensors and actuators. Review on using piezoelectric materials to MEMS sensor [1], morphing aircraft [2], and structural repair [3] have been reported. Yang and Chiu [4] were among the first to embedded piezoelectric sensors inside composite-laminated structures. The sensors were found to have stiffening effects [5-8]. Among the applications; however, piezoelectric sensor measurement was considered as displacement signal [9-11], velocity signal $[12,13]$, or strain rate signal $[14,15]$. There seems to be inconsistency on the signal nature, and signal conditioning circuit is often necessary.

It is known that effective vibration control requires the system state of displacement and velocity; however, such signals are difficult to acquire as they are often obtained either by accelerometer with hardware integration for velocity or by piezoelectric sensor assuming velocity measurement. Accurate sensor dynamics modeling is required for designing a controller immune to modeling discrepancy. Artificial neural networks with the ability of self-learning, generalization, and robustness have been shown suitable for simulating sensor dynamics by system identification. The concept of neural sensor design is to use the piezoelectric sensor measurement to estimate online both the displacement and velocity at the sensor location. Recent development of smart layer by encapsulating piezoelectric sensor, actuator, and controller [13] has demonstrated the dissemination of piezoelectric sensor to many engineering applications, and the need of simulating displacement and velocity signal becomes evident.

\section{Neural Sensor}

Traditional system identification emphasizes on parameter estimation in which the configuration of system model must be defined beforehand. The neural network in this study does not intend to identify the system parameters (such as mass, damping ratio, and stiffness), but instead to find the connection weights of neural network through the experimental input/output pairs so that a piezoelectric sensor, 


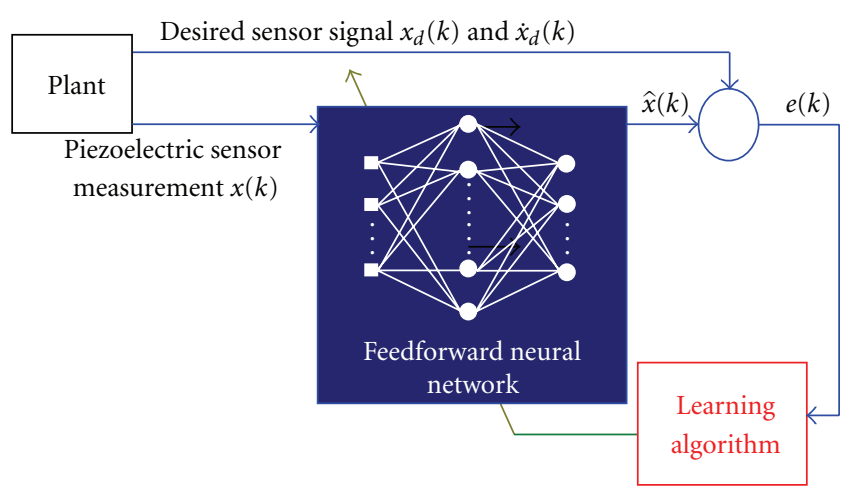

Figure 1: A feedforward neural network with learning algorithm to simulate the desired displacement and velocity signals by piezoelectric sensor measurement.

whether embedded or surface attached, can faithfully acquire the displacement and velocity. In simulating sensor dynamics by neural network, the input and output can be any physical parameters accessible experimentally. Figure 1 illustrates the neural sensor design by using the feedforward network to simulate the desired displacement and velocity signals $x_{d}(k)$ and $\dot{x}_{d}(k)$ from the piezoelectric sensor measurement $x(k)$. The error $e(k)$ between the desired signals and the network output $\hat{x}(k)$ is then minimized by the learning algorithm.

Artificial neural network is an information process with neurons to imitate the system state of both displacement and velocity by using the piezoelectric sensor measurement. The relationship between the input and output pattern of an artificial neuron can be represented by $h_{j}=\sum_{i} w_{i j} x_{i}-\theta_{j}$ and $y_{j}=f\left(h_{j}\right)$ where $x_{i}$ is the input; $\theta_{j}$ is the bias representing the threshold of the transfer function; $w_{i j}$ is the connection weight for imitating the biological synapse strength; $h_{j}$ is the linear combination; $y_{j}$ is the output; $f\left(h_{j}\right)$ is the transfer function (or the activity function) for converting the weighted summation from the input to output. There are two neural network structures: the feedforward neural network and the feedback neural network. In the former the output of a neuron is passed only to the next layer but not to the preceding layer(s) or to the other neurons in the same layer, while in the latter the output is sent back to the neurons of the preceding layer(s). A feedforward network model may have many layers, and each layer contains several processing neurons as illustrated in Figure 1. The hidden layer provides the neuron interactions representing the system's internal structure.

The error backpropagation algorithm is commonly used in training a neural network. Recent study on the performance of different algorithms showed that the algorithm is a first-order approximation of the steepest descent technique based on the gradient of the instantaneous error [16]. The update rule for the connection weight at time step $(k+1)$ is defined as

$$
\Delta w_{i j}(k+1)=-(1-\alpha) \eta(k+1) \frac{\partial E}{\partial w_{i j}}+\alpha \Delta w_{i j}(k)
$$

where $\alpha$ is a user-selected positive momentum constant $(0<$ $\alpha<1)$ to allow the network to respond not only to the local gradient but also to recent trends in the error, $\eta(k+1)$ is the learning rate at step $(k+1)$, and $E$ is the error function defined by the square of the error between the desired output and the neuron output. A neural network model is inherently nonlinear because the transfer function of the hidden layer neuron is often in sigmoid function or hyperbolic tangent function. The neural sensor can be described in the form

$$
\begin{gathered}
y(k+1)=F[y(k), y(k-1), \ldots, y(k-n+1), u(k), \\
u(k-1), \ldots, u(k-m+1)],
\end{gathered}
$$

where $[u(k), y(k)]$ represents the input/output pair at time step $k, m$ is the maximal lags in the input, $n$ is the maximal lags in the output, and $F[\cdot]$ represents the nonlinear input/output transformation. The output at time step $(k+1)$ depends both on its past $n$ steps of the output $y(k-i)$, $i=0,1, \ldots, n-1$, and the past $m$ steps of the input $(k-j)$, $j=0,1, \ldots, m-1$. The estimated output $\hat{y}$ at time step $(k+1)$ is

$$
\begin{gathered}
\hat{y}(k+1)=F[y(k), y(k-1), \ldots, y(k-n+1), u(k), \\
u(k-1), \ldots, u(k-m+1)],
\end{gathered}
$$

which is known as the series-parallel identification model, often more useful in generating a stable update rule. By comparison, the parallel model represented by

$$
\begin{gathered}
\hat{y}(k+1)=F[\hat{y}(k), \hat{y}(k-1), \ldots, \hat{y}(k-n+1), u(k), \\
u(k-1), \ldots, u(k-m+1)]
\end{gathered}
$$

is to validate the network effectiveness in identification. System identification contains two phases: the network learning phase and the validation phase. The process is to find the appropriate connection weights by the learning algorithm until the error between the network output and the desired output is small enough, then to validate if the network output $\hat{y}(k)$ is close to the actual output $y(k)$.

\section{Vibration Control Application}

Effectiveness of the neural sensor is demonstrated by vibration contral of a smart structure, a $[90 / 90 / 90 / 90 / 0]_{\times 2}$ composite-laminated beam of $265 \times 40.5 \times 1 \mathrm{~mm}$ in Sglass/epoxy unidirectional pre-prag tapes (Fiberite HyE9134B), with two embedded $120 \times 30 \times 0.375 \mathrm{~mm}$ piezoelectric actuators and one $13.5 \times 25 \times 0.375 \mathrm{~mm}$ piezoelectric sensor. Mechanical properties of uni-directional laminate, and the electro-mechanical properties of piezoelectric material are listed in Tables 1 and 2, respectively, where piezoelectric material is for both sensing and actuation due to its low-temperature sensitivity, high strain sensitivity, and fast response. Experiments as shown in Figure 2 are conducted on the smart structure under clamp-free boundary condition. One piezoelectric actuator driven by an independent and identically distributed random signal is employed to excite the structure, and the piezoelectric sensor 


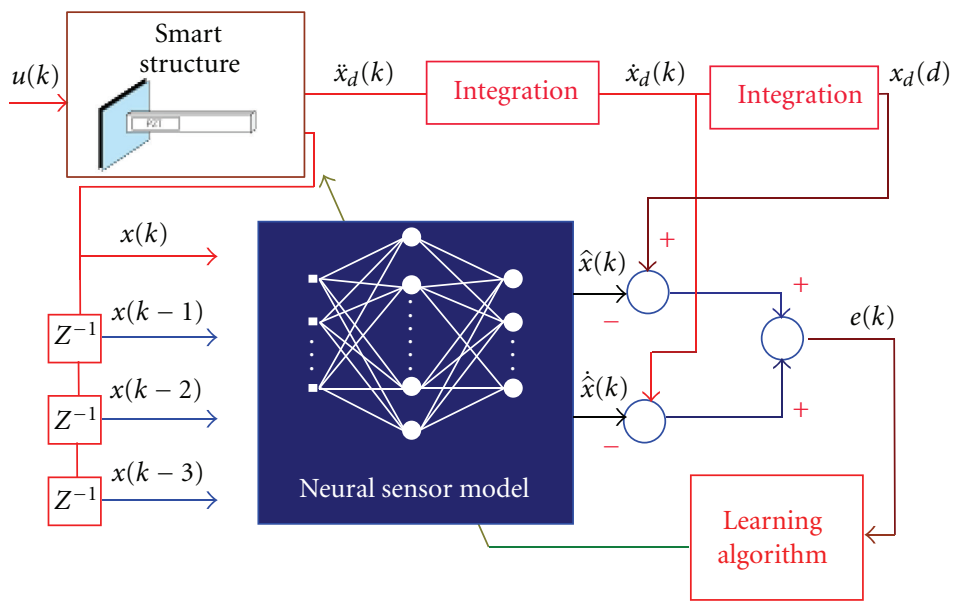

FIGURE 2: Application of the piezoelectric neural sensor to simulate the displacement and velocity signals of a smart structure.
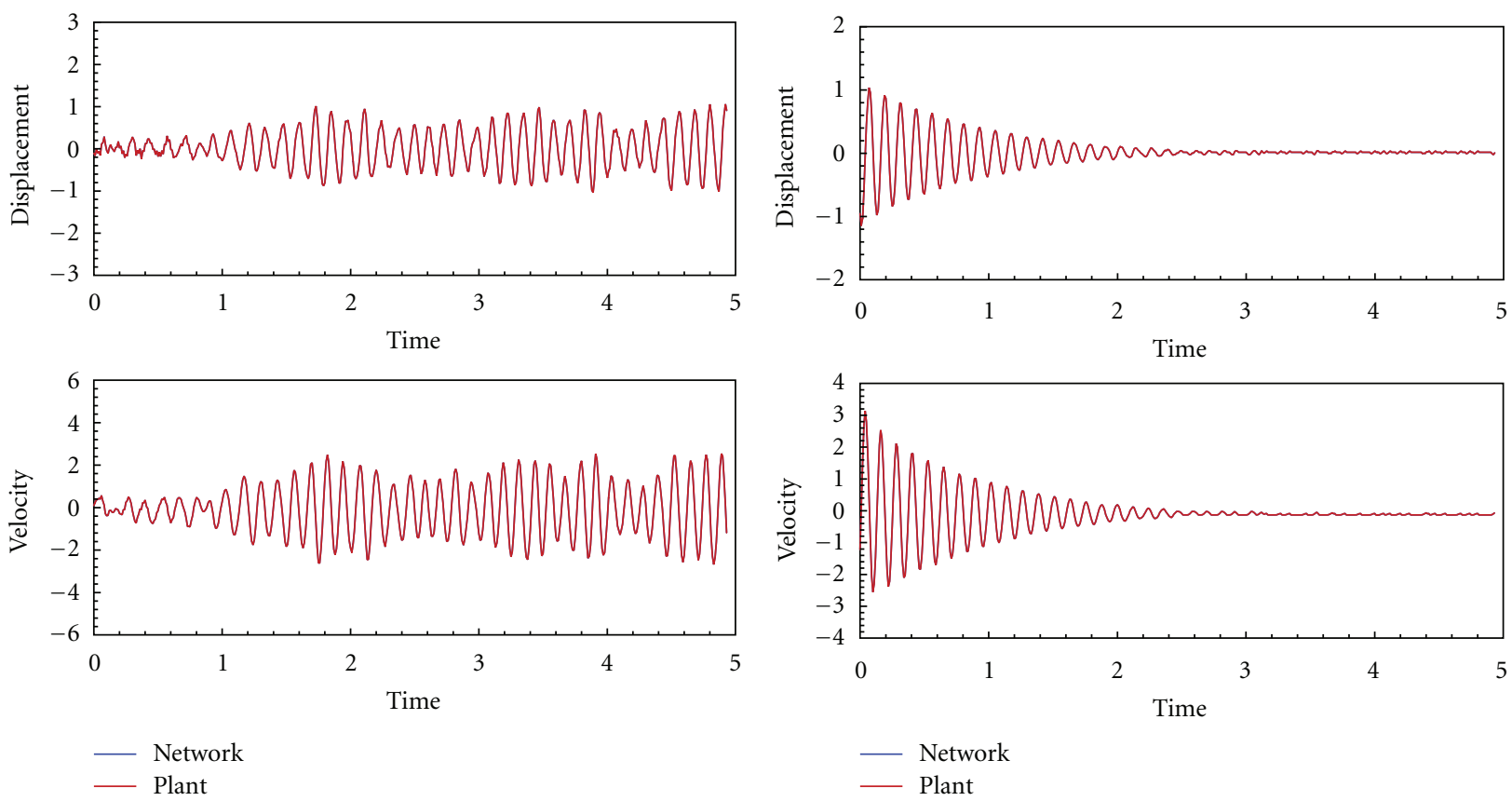

(a)

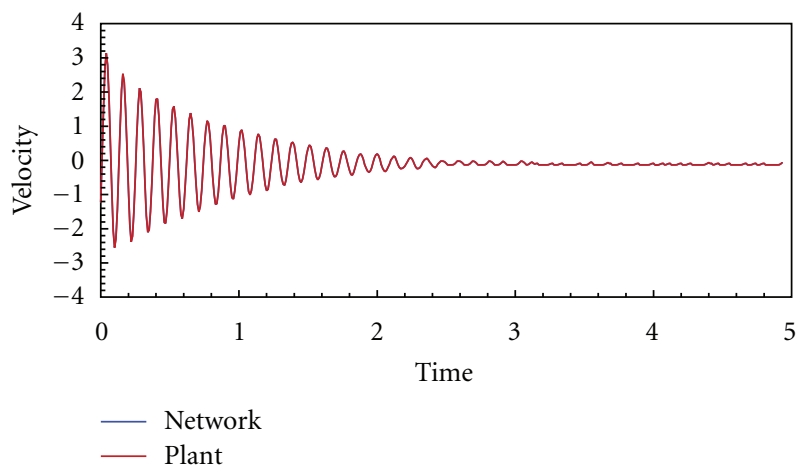

(b)

FIGURE 3: Experimental results of the neural sensor signal (Network) and the accelerometer signal (Plant) in the smart structure under (a) random excitation and (b) free vibration. The discrepancies in both displacement and velocity signals are negligible.

embedded near the clamped end and the accelerometer (B\&K 4375) attached at the beam tip are sampled at $100 \mathrm{~Hz}$. The measurement of accelerometer is then integrated to yield velocity and displacement signals, $\dot{x}_{d}(k)$ and $x_{d}(k)$. A total of 2000 data points is collected for training the neural sensor.

Analyses show that using the previous four steps of the sampled sensor signals as the neural network's input can give reasonably accurate estimate of displacement and velocity. The neural network structure is of $[4 * 3 * 2]-4$ neurons in the input layer, 3 in the hidden layer, and 2 in the output layer. The number of neurons the hidden layer is selected to be adequately small because more neurons do not necessarily yield better results for they may increase the learning time and complexity. Comparison of the time response between
TABLE 1: Mechanical properties of the uni-directional laminates (Sglass/epoxy).

\begin{tabular}{lc}
\hline Elastic modulus $E_{1}$ & $55.55 \mathrm{GPa}$ \\
Elastic modulus $E_{2}$ & $25.9 \mathrm{GPa}$ \\
Elastic modulus $G_{12}$ & $7.7 \mathrm{GPa}$ \\
Elastic modulus $G_{13}$ & $7.7 \mathrm{GPa}$ \\
Poisson ratio $\nu_{12}$ & 0.26 \\
Density & $1881 \mathrm{~kg} / \mathrm{m}^{3}$ \\
\hline
\end{tabular}

the desired displacement and velocity signals and the neural sensor is shown in Figure 3(a), in which the neural sensor is so accurate in simulating both the displacement and velocity. 


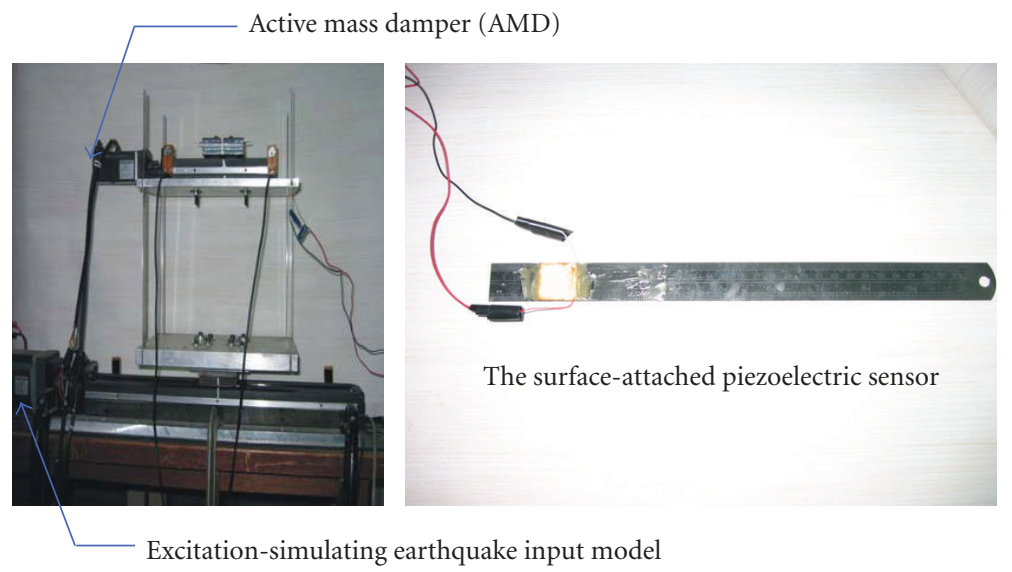

(a)

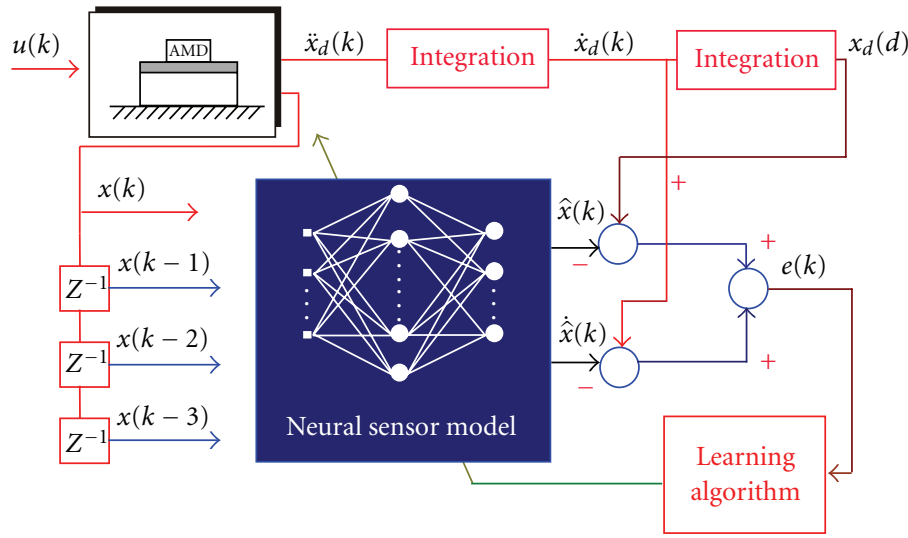

(b)

FIGURE 4: Application of the neural sensor to simulate the displacement and velocity signals in the structural control experiment with active mass damper (AMD).

TABLE 2: Electromechanical properties of the piezoelectric material.

\begin{tabular}{lc}
\hline Density & $7.6 \mathrm{~g} / \mathrm{cc}$ \\
Young's modulus & $6.8 \mathrm{~N} / \mathrm{m}^{2} \times 10^{10}$ \\
Curie temperature & $340^{\circ} \mathrm{C}$ \\
Mechanical $Q$ & 400 \\
Energy conversion $K_{31}$ & $35 \%$ \\
Piezoelectric coefficient $d_{31}$ & $-125 \times 10^{-12} \mathrm{~m} / \mathrm{V}$ \\
Piezoelectric coefficient $d_{33}$ & $300 \times 10^{-12} \mathrm{~m} / \mathrm{V}$ \\
\hline
\end{tabular}

Another validation as shown in Figure 3(b) is conducted by comparing the free vibration response under the same initial displacement. The neural sensor is again shown faithfully simulating the displacement and velocity.

In addition to the smart structure, a one-story shearbuilding structure model of $300 \times 210 \times 205 \mathrm{~mm}$ and weights $5.4 \mathrm{~kg}$ with an active mass damper (AMD) mounted on the top floor as shown in Figure 4(a) [17] is also applied to validate the neural sensor effectiveness. The structure mass is concentrated at the floor level, and the columns flexible to lateral deformation but rigid in vertical direction are assumed to be mass less. Its natural frequency is in the range of common high-rise buildings at about $1.6 \mathrm{~Hz}$. Two AC servomotors (Mitsubishi HC-MF13 and MF73) as listed in Table 3 are used: one for driving the mass damper mounted on the linear guideway (THK KR33) with a moving part $1 \mathrm{~kg}$ at maximum stroke $\pm 50 \mathrm{~mm}$ and the other for driving the building structure at the ground floor to simulate earthquake excitation. A $13.5 \times 25 \times 0.375 \mathrm{~mm}$ piezoelectric sensor is attached on a column of the building model, and a low-frequency accelerometer (Kistler 8628B5) is attached at the top floor to acquire the acceleration signal. The servomotor driven by an independent and identically distributed random signal is employed to excite the structure, and the measurements of the attached accelerometer and piezoelectric sensor signal are sampled at $10 \mathrm{~Hz}$.

The neural sensor is a $[4 * 5 * 2]$ feedforward neural network. The learning pattern consists of 931 input/output pairs, and the training goes until the sum-squared error is small within a predefined threshold. Validation of the earthquake response between the accelerometer and neural sensor is shown in Figure 5(a) in which the neural sensor is so accurate in simulating both the displacement and velocity signals. Another validation is conducted by comparing the free vib- 

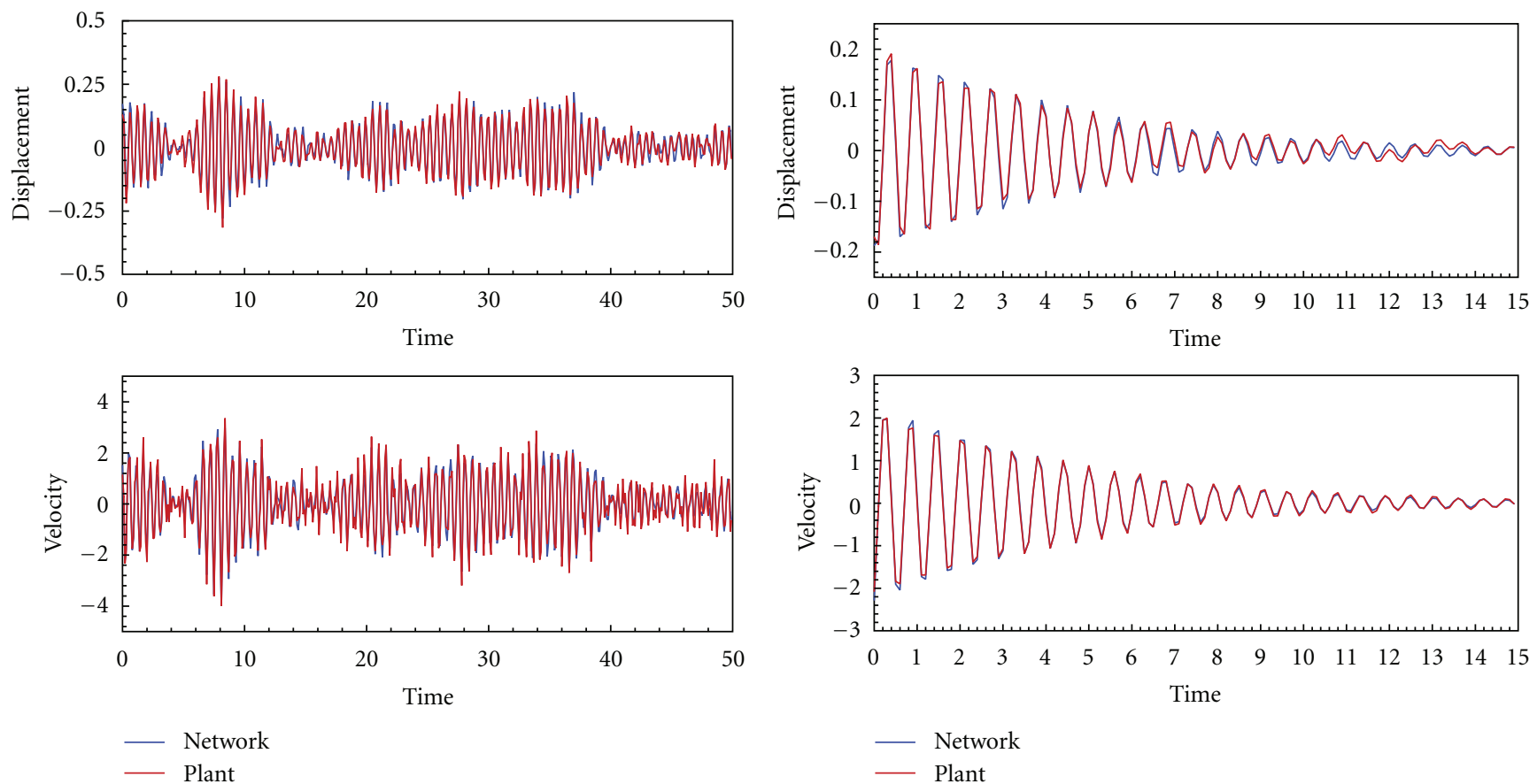

(a)

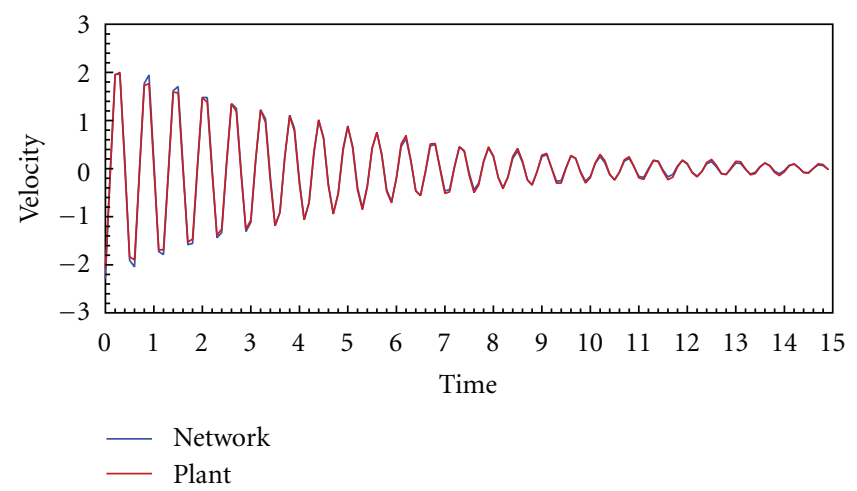

(b)

FIGURE 5: Experimental result of the neural sensor signal (Network) and the accelerometer signal (Plant) in the structure model under (a) random excitation and (b) free vibration. The discrepancies in both displacement and velocity signals are negligible.

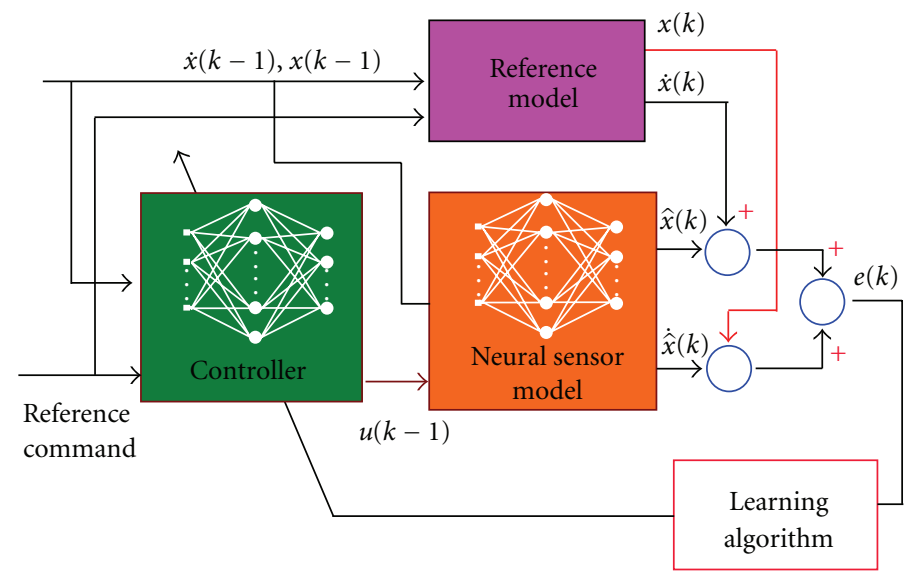

FIGURE 6: Schematic diagram of the neural controller design.

ration response as shown in Figure 5(b). The discrepancy appears only at the peak vibration amplitude, and the performance of the neural sensor remains excellent.

Effective controller design for vibration suppression can be facilitated by having accurate system state of displacement and velocity measurement. The neural network controller design is shown in Figure 6. All that required is a reference model for the desired system performance. The neural controller can be self-organized online by using the discrepancy between the plant and the reference model. From experimental observation, the first vibration mode of the smart structure is usually dominant over the other so that a secondorder reference model for the smart structure is selected as

$$
\ddot{x}+5 \dot{x}+2402 x=2402 u \text {. }
$$

The damping ratio is limited by the controller output voltage of $\pm 10 \mathrm{~V}$. A [ $3 * 3 * 1$ ] feedforward neural network that is constructed to train the controller. The transfer function of the hidden layer is hyperbolic tangent function and that of the output layer is linear function. The input of the neural network controller is displacement and velocity of the neural 


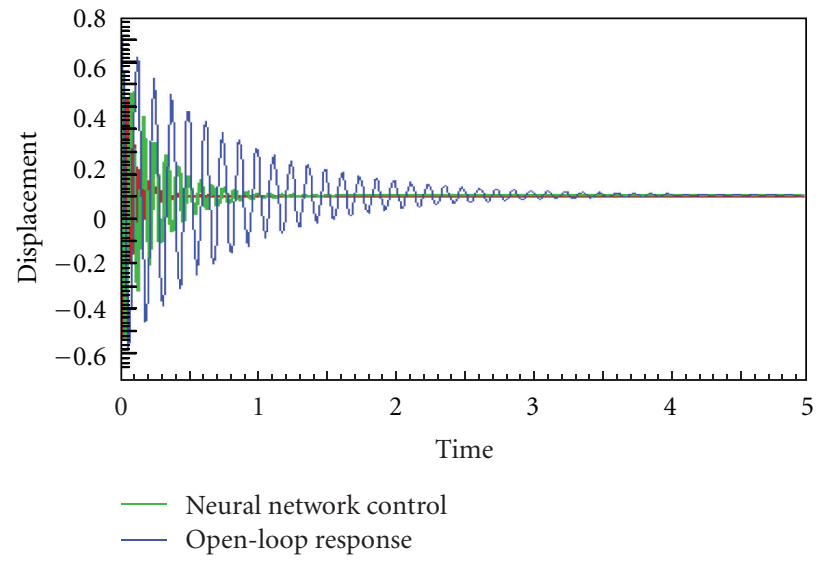

(a)

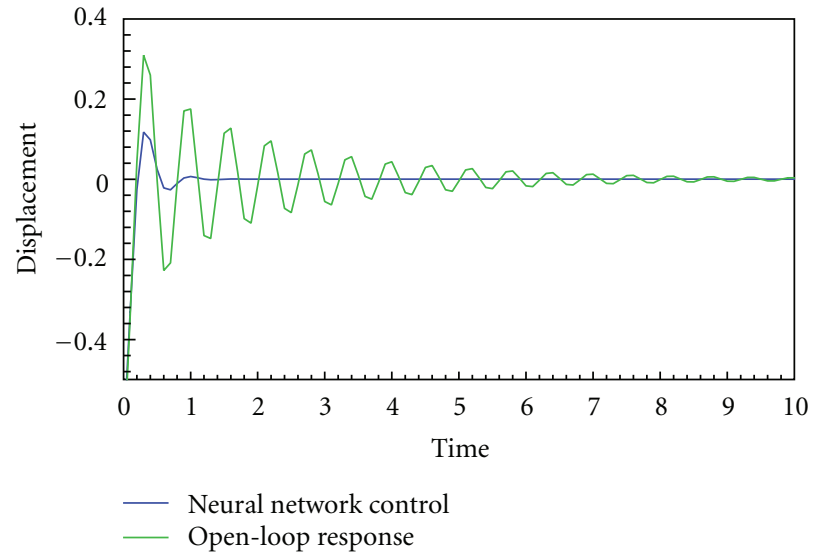

(b)

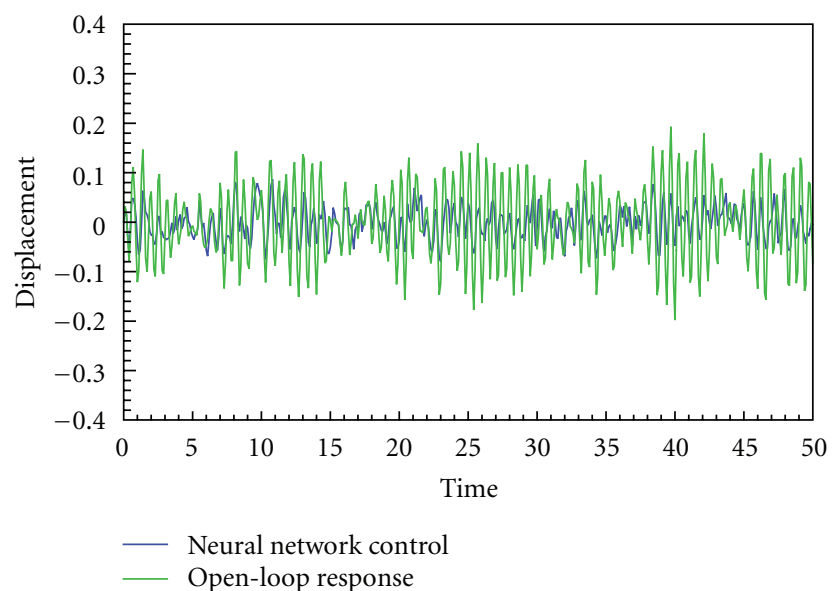

(c)

FIGURE 7: Open- and closed-loop experimental results of (a) the smart structure by the neural sensor and controller, and the response of the building structure under (b) free vibration and (c) earthquake excitation by the neural sensor and controller.

TABle 3: Specification of the AC servomotor.

\begin{tabular}{lcc}
\hline \multirow{2}{*}{ Item } & \multicolumn{2}{c}{ Servo Motor } \\
& HC-MF13 & HC-MF73 \\
\hline Rated output (kW) & 0.10 & 0.75 \\
Rated torque (Nm) & 0.32 & 2.40 \\
Maximum torque (Nm) & 0.95 & 7.20 \\
Rated speed (rpm) & \multicolumn{2}{c}{3000} \\
Maximum speed (rpm) & \multicolumn{2}{c}{4500} \\
Permissible instantaneous speed (rpm) & \multicolumn{2}{c}{5175} \\
Inertia moment $\left(\mathrm{kgcm}^{2}\right)$ & 0.03 & 0.60 \\
Weight (kg) & 0.53 & 3.00 \\
\hline
\end{tabular}

sensor and the controller command, and the desired output is calculated from (5). The training pattern consists of 1000 input/output pairs.

One piezoelectric actuator is employed to excite the smart structure, and the system state from the neural sensor is sampled at $100 \mathrm{~Hz}$. The neural controller is a [3* $3 * 2$ ] feedforward neural network with three input: the system displacement, velocity, and excitation force, $x(k)$, $\dot{x}(k)$, and $u(k)$, and two output $\hat{x}(k+1)$ and $\dot{\hat{x}}(k+1)$. A voltage amplifier (Krohn-Hite 7500) is employed to drive the actuator for better control performance. Figure 7(a) shows the free vibration response of the smart structure, where the residual vibration from an initial disturbance is reduced from 4 seconds to within 1 second. The experimental result indicates that the neural sensor is effective in facilitating the neural controller in vibration suppression.

The neural controller is also applied to the building control experiment. The schematic diagram of the controller design is similar to Figure 6. From experimental observation, the reference model is chosen as a second-order system

$$
\ddot{x}+5 \dot{x}+110.1 x=101.1 u,
$$

in which the damping coefficient is selected to be higher than that of the uncontrolled plant. A [3*3*1] feedforward neural network, similar to that of smart structure, is used for training the controller with three input neurons: the displacement and velocity of the neural sensor and the controller command. The 1000 input/output pairs for training the controller are chosen in the vibration range of 
the building structure, and the desired output set is calculated from (6). The servomotor is employed to excite the structure, and the system state from the neural sensor is sampled at $10 \mathrm{~Hz}$. Figure $7(\mathrm{~b})$ is the free vibration response of the building structure under an initial displacement at the top floor in which the settling time of the open-loop system is more than 7 seconds whereas that of the closed-loop system is less than 1 second. The residual vibration is suppressed effectively. Figure 7(c) also shows the vibration response of the open and closed loop of the building structure subject to an earthquake excitation. The above experimental results show that satisfactory performance can be achieved by the neural sensor in simulating the displacement and velocity signals and by the neural network controller in providing the desired damping effect.

\section{Conclusions}

(1) Intelligent structures with built-in piezoelectric sensor and actuator have been known preferable in vibration control; however, there seems to be inconsistency on the signal nature of piezoelectric sensor. It is often arguable to determine if measurement of piezoelectric sensor is strain rate, displacement, or velocity signal. A neural sensor to simulate the sensor dynamics is developed by an artificial neural network so that the embedded or attached piezoelectric sensor can faithfully measure the displacement and velocity without any signal conditioning circuitry. Experimental verification shows that the neural sensor is effective in vibration suppression of a smart structure by embedded sensor/actuator and a building structure by a surface-attached piezoelectric sensor with active mass damper.

(2) A neural sensor based on a [4*3*2] feedforward neural network identification model is developed to identify successfully the sensor dynamics of a smart structure, while another $[4 * 5 * 2]$ model has also been applied to a building structure system. The neural sensors are shown to faithfully simulating the displacement and velocity of the systems in both free vibration and under random excitation. A threelayer neural controller is then developed for vibration control of building structure and smart structure. It should be noted that no assumption is required in training the controllers. Experimental results show that the controller design based on the neural sensor's displacement and velocity signals is effective to vibration suppression.

\section{Acknowledgments}

This work was supported in part by the National Science Council, Taiwan, ROC under NSC100-2221-E006-098MY3.

\section{References}

[1] S. Tadigadapa and K. Mateti, "Piezoelectric MEMS sensors: state-of-the-art and perspectives," Measurement Science \& Technology, vol. 20, no. 9, Article ID 092001, 2009.

[2] S. Barbarino, O. Bilgen, R. M. Ajaj, M. Friswell, and D. J. Inman, "A review of morphing aircraft," Journal of Intelligent Material Systems and Structures, vol. 22, no. 9, pp. 823-877, 2011.

[3] Q. Wang and N. Wu, "A review on structural enhancement and repair using piezoelectric materials and shape memory alloys," Smart Materials and Structures, vol. 21, no. 1, Article ID 013001, 2012.

[4] S. M. Yang and J. W. Chiu, "Smart structures_-vibration of composites with piezoelectric materials," Composite Structures, vol. 25, no. 1-4, pp. 381-386, 1993.

[5] S. M. Yang and Y. J. Lee, "Interaction of structure vibration and piezoelectric actuation," Smart Materials and Structures, vol. 3, no. 4, pp. 494-500, 1994.

[6] S. M. Yang and J. J. Bian, "Vibration suppression experiments on composite laminated plates using an embedded piezoelectric sensor and actuator," Smart Materials and Structures, vol. 5, no. 4, pp. 501-507, 1996.

[7] S. M. Yang and C. W. Chen, "Application of single mode optical fiber sensors in structural vibration suppression," Journal of Intelligent Material Systems and Structures, vol. 7, no. 1, pp. 71-77, 1996.

[8] S. M. Yang and J. A. Jeng, "Vibration control of a composite plate with embedded optical fiber sensor and piezoelectric actuator," Journal of Intelligent Material Systems and Structures, vol. 8, no. 5, pp. 393-400, 1997.

[9] S. M. Yang and G. S. Lee, "Vibration control of smart structures by using neural networks," Journal of Dynamic Systems, Measurement and Control, vol. 119, no. 1, pp. 34-39, 1997.

[10] S. M. Yang and G. S. Lee, "System identification of smart structures using neural networks," Journal of Intelligent Material Systems and Structures, vol. 8, no. 10, pp. 883-890, 1997.

[11] H. Irschik, "A review on static and dynamic shape control of structures by piezoelectric actuation," Engineering Structures, vol. 24, no. 1, pp. 5-11, 2002.

[12] C. A. Jeng, S. M. Yang, and J. N. Lin, "Multi-mode control of structures by using neural networks with marquardt algorithms," Journal of Intelligent Material Systems and Structures, vol. 8, no. 12, pp. 1035-1043, 1997.

[13] S. M. Yang, C. C. Hung, and K. H. Chen, "Design and fabrication of a smart layer module in composite laminated structures," Smart Materials and Structures, vol. 14, no. 2, pp. 315-320, 2005.

[14] S. M. Yang and C. A. Jeng, "Structural vibration suppression by concurrent piezoelectric sensor and actuator," Smart Materials and Structures, vol. 5, no. 6, pp. 806-813, 1996.

[15] J. Zhao, J. Tang, and K. W. Wang, "Anomaly amplification for damage detection of periodic structures via piezoelectric transducer networking," Smart Materials and Structures, vol. 20, no. 10, Article ID 105006, 2011.

[16] C. L. Su, S. M. Yang, and W. L. Huang, "A two-stage algorithm integrating genetic algorithm and modified Newton method for neural network training in engineering systems," Expert Systems with Applications, vol. 38, no. 10, pp. 12189-12194, 2011.

[17] S. M. Yang, C. J. Chen, and W. L. Huang, "Structural vibration suppression by a neural-network controller with a massdamper actuator," Journal of Vibration and Control, vol. 12, no. 5, pp. 495-508, 2006. 

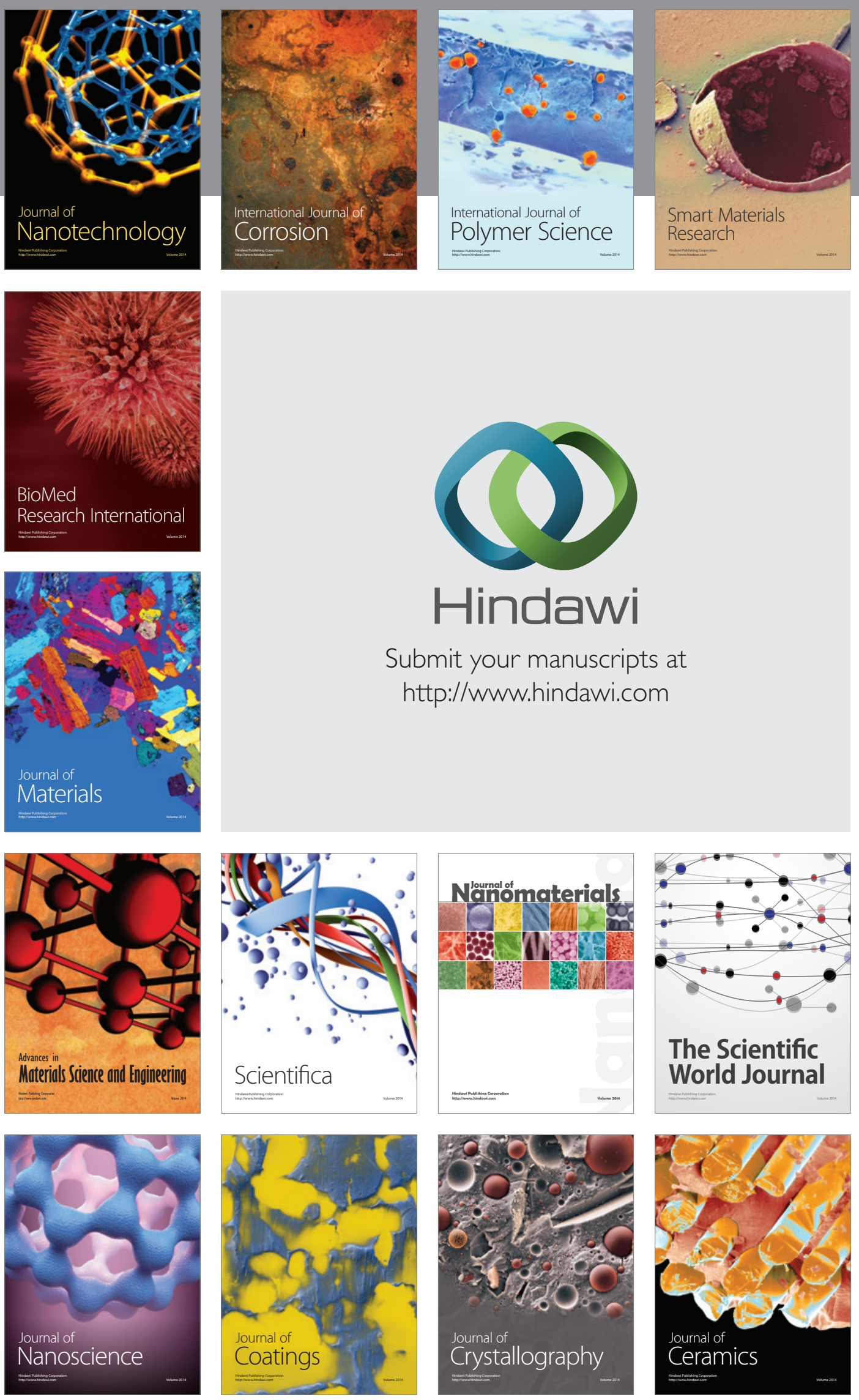

The Scientific World Journal

Submit your manuscripts at

http://www.hindawi.com

\section{World Journal}

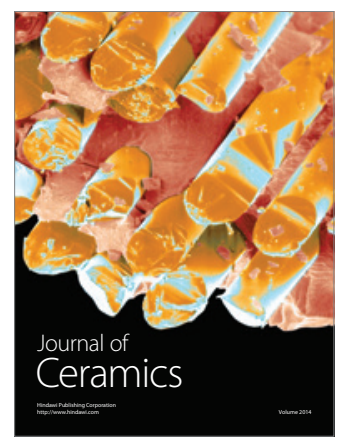

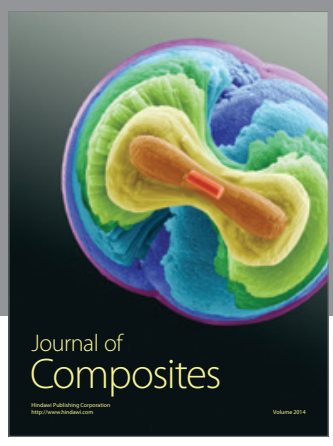
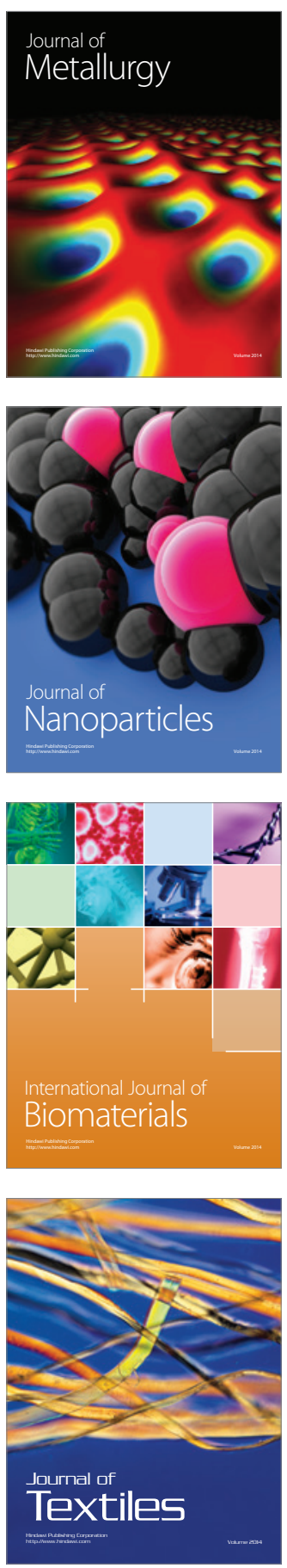